\title{
A Comparative Study of Cultured Smooth Muscle Cell Proliferation and Injury, Utilizing Glycated Low Density Lipoproteins with Slight Oxidation, Auto-oxidation, or Extensive Oxidation
}

\author{
Seiji Taguchi, Toshinori Oinuma, and Tsutomu Yamada \\ Department of Pathology, Nihon University, School of Medicine, Tokyo, Japan.
}

\begin{abstract}
We investigated the influence of glycated low density lipoprotein (LDL) for vascular smooth muscle cell (SMC) proliferation or injury. We utilized glycated, slightly oxidized LDL (GLDLLOX), glycated, auto-oxidized LDL (GLDL) and glycated, metal-induced extensively oxidized LDL (GLDL-OX) to examine the effect of glycation itself or combined glycation and oxidation on SMC. GLDL-LOX induced SMC proliferation and migration, and increased the number of platelet-derived growth factor receptor, $\beta$ subunits, (PDGF-R) positive SMC. Also, GLDLLOX promoted protease activity, compared with the other groups including native LDL (control). GLDL and GLDL-OX demonstrated SMC injury with apoptosis and Bax protein expression, compared with native LDL and GLDL-LOX. These results suggested that LDL glycation contributed to the progression of atherosclerosis by promoting SMC migration and proliferation, with little dependence on oxidative modification. Secondary auto-oxidation adding to glycation induced SMC apoptosis, and SMC injury occurred in the state of strong oxidation with glycation. We concluded that LDL glycation might play a key role in the progression of atherosclerosis in diabetes, and glycated LDL promoted atherosclerosis, even with little assistance from oxidation. $J$ Atheroscler Thromb, $2000 ; 7: 132-137$.
\end{abstract}

Key words : Glycated LDL, Oxidative stress, Smooth muscle cells, Atherosclerosis

\section{Introduction}

Diabetes mellitus is well-known to accelerate atherosclerotic lesions as well as microvascular injury (1). Even in young people, high glycohemoglobin group (diabetic) raises the atherosclerotic lesions more extensively than normal subjects (non-diabetic) (2). It is still unclear how hyperglycemia affects the atherosclerosis progression.

The modified low density lipoprotein (LDL) is unanimously recognized as an important factor for the progression of atherosclerosis $(3,4)$. LDL particles in diabetic patients might be altered in a number of ways that may affect their metabolism and atherogenecity $(5,6)$. These mainly include alteration in composition, enhanced

Address for correspondence: Seiji Taguchi, Department of Pathology, Nihon University, School of Medicine 30-1, Oyaguchikamicho, Itabashi-ku, Tokyo 173-8610, Japan.

Tel: +81-3-3972-8111 int2256, Fax: +81-3-3972-8163

Received November 5, 1999.

Accepted for publication June 30, 2000 nonenzymatic glycosylation (5) and increased susceptibility to oxidative modification $(7,8)$. Glycated LDL is increased in diabetic patients, where disease also proceeds under euglycemic conditions (9). The function of glycated LDL, especially glycated and slightly oxidized LDL, has neither been explained well nor distinguished from secondarily oxidized LDL in diabetes.

In this study, we utilized three glycated LDLs; 1. glycated and slightly-oxidized LDL 2. glycated and autooxidized LDL, and 3. glycated and metal-induced extensively-oxidized LDL. The effect of these glycated LDLs for vascular smooth muscle cell (SMC) was investigated in order to clarify the influence of glycated LDL on atherosclerosis. Our study was focused on the proliferation, migration, cell injury, programmed cell death (apoptosis) and protease activity of SMC influenced by three different types of glycated LDL addition. 


\section{Materials and Methods}

\section{Low density lipoprotein (LDL)}

LDL, derived from human plasma, was purchased from Sigma Co., St. Louis, USA LDL was used immediately after purchase as native LDL ( $n L D L)$. This purchased LDL was lyophilized from $1 \mathrm{mM}$ of LDL solution in $0.15 \mathrm{M}$ $\mathrm{NaCl}$ and $0.01 \%$ EDTA at $\mathrm{pH} 7.4$ and refrigerated at $4^{\circ} \mathrm{C}$.

\section{LDL preparations}

LDL was incubated with $200 \mathrm{mM}$ glucose at $37^{\circ} \mathrm{C}$ for 1 weeks (10). This LDL was indicated as glycated LDL (GLDL), which seemed to be naturally and minimally oxidized LDL. Butyl-hydroxytoluene (BHT : $25 \mu \mathrm{g} / \mathrm{I})$ and EDTA were added to LDL at the starting point of incubation with glucose, in order to stop LDL oxidation. The LDL produced by this method was called glycated LDL with slight oxidation (GLDL-LOX). LDL incubated with $200 \mathrm{mM}$ glucose for 1 week with $8 \mu \mathrm{mol} \mathrm{CuSO}_{4}$ for 48 hours to produce extensively-oxidized, glycated LDL, was indicated as GLDL-OX. The thiobarbituric acid-reactive substances (TBARS) values of each utilized LDL were follows ; nLDL : $0.11 \pm 0.08$, GLDL-LOX : $0.16 \pm 0.01$, GLDL : $0.22 \pm 0.04$, GLDL-OX : $0.4 \pm 0.22$ (nmol/100 $\mu \mathrm{g}$ protein). There was a significant difference between the TBARS value of $n L D L$ and that of GLDL-OX $(p<0.05)$. The lag time for conjugated diene formation was $100 \pm 20$ minutes in GLDL-OX. In the other three groups, the lag time exceeded 180 minutes.

\section{Cell culture}

SMC was purchased from Kurabou Co. (Kurashiki, Japan), derived from media of human aorta, twice-passaged $\left(5 \times 10^{5} / \mathrm{ml}\right)$. The conditions of cell culture were $37^{\circ} \mathrm{C}, 5 \% \mathrm{CO}_{2}$. These cells were divided and seeded in the slide chamber (Nunc Co. Naperville, USA), and filled with medium without any serum. The cells were incubated with (a) nLDL : $n=5$, (b) GLDL-LOX : $n=5$, (c) GLDL : $n=5$, and (d) GLDL-OX; $n=5$, in each chamber space for 24 hours.

\section{Cell number count}

After incubation in a slide chamber, each slide was fixed in neutral buffered formalin, and stained with hematoxylin solution. The cell number of each chamber was counted. Each datum was expressed as the percentage of cell number before incubation.

\section{SMC migration rate}

SMC were divided and seeded into a chemotaxis chamber (Kurabo Co., Japan). Each chemotaxis was put into 24 wells filled with medium. The cells which migrated to the lower surface of chemotaxis were counted. Each datum was expressed as the percentage of preincubation SMC number.

\section{Apoptosis}

Apoptotic cells were examined by the TUNEL method, using an ApopTag (Oncor, Gaithersburg, USA) kit for probing DNA fragmentation. Briefly, each slide was fixed with $10 \%$ neutral buffered-formalin. After quenching endogenous peroxidase, an equilibration buffer was applied. Then, terminal deoxynucleotidyl transferase (TdT) was applied, followed by prewarmed Stop/Wash buffer at $37^{\circ} \mathrm{C}$ for 1 hour. Finally, anti-digoxygenin-peroxidase was added. Diaminobenzidine tetrahydrochloride (DAB) was utilized for color development. Slides were counter-stained and mounted. Data were expressed as the percentage of total cell number.

\section{Immunohistochemistry for platelet-derived growth factor receptor (PDGF-R) and Bax protein}

Each slide was fixed with $10 \%$ neutral buffered formalin. Briefly, each slide was incubated with mouse antihuman PDGF-R, $\beta$ subunit, monoclonal antibody (Genzyme Co., Cambridge, USA ; dilution $1: 100)$ and Bax protein monoclonal antibody (MBL Co., Nagoya, Japan ; dilution 1 : 100) at $4^{\circ} \mathrm{C}$ overnight after blocking by normal serum. After washing, the slide was incubated with the second antibody at room temperature, followed by incubation with avidin-biotin complex at room tempreture. Immunoreactivity of PDGF-R and Bax was developed by DAB. The positive cells were counted, and data were expressed as the percentage of total cell number.

\section{Protease activity}

The gel was prepared as follows; $1 \%$ agarose melted in $50 \mathrm{mmol} / \mathrm{I}$ Tris- $\mathrm{HCl}, \mathrm{pH} 7.4$, containing $10 \mathrm{mmol} / \mathrm{I}$ calcium chloride and $0.05 \%$ Brij 35 was mixed $1: 1$ with $1 \mathrm{mg} / \mathrm{ml}$ casein (11). The supernatant of each group was mixed with this gel. After reaction for 1 month, the weight of each gel was calculated. Each datum was expressed as follows ; $100-100 \times$ Gel weight after reaction $/$ Gel weight before reaction (\%).

\section{Statistical evaluation}

Results are expressed as mean \pm S.D. of the overall data. The statistical significance of the difference between various parameters was calculated using the students $t$ test. The significant difference was taken as $p<0.05$.

\section{Results}

\section{SMC number}

The number of SMC incubated with GLDL-LOX was significantly increased $(p<0.05)$ compared with the $n L D L$. This revealed that GLDL-LOX might promote SMC proliferation. On the other hand, the SMC number incubated with GLDL-OX was significantly decreased $(p<0.05)$ compared with the nLDL. This indicated that GLDL-OX induced SMC cytotoxicity. There was no significant 


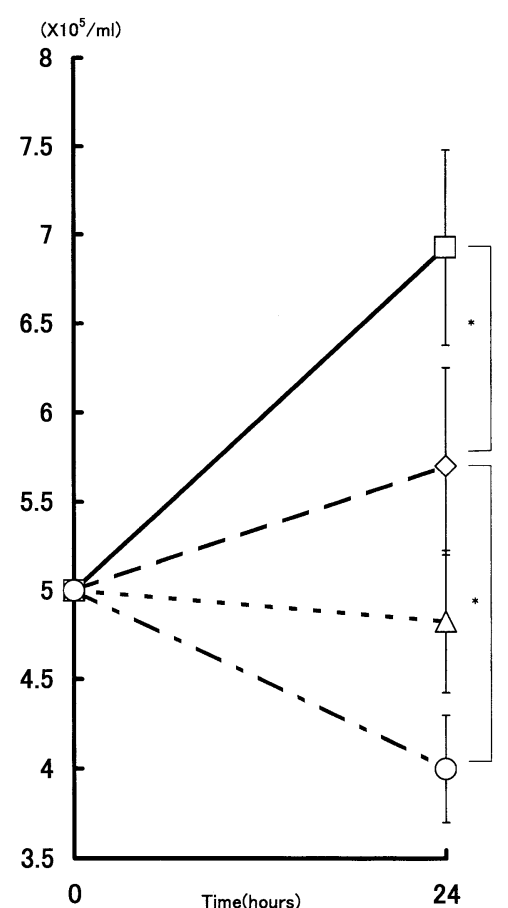

Fig. 1. This graph showing the change of SMC number between before incubation and 24 hours after incubation. Cell number before incubation is $5 \times 10^{5} / \mathrm{ml}$, each group. The value of GLDL-LOX group is higher, and the value of GLDL-OX is lower than that of nLDL (control) group, after 24 hours.

$\diamond:$ nLDL group, $\square:$ GLDL-LOX group, $\triangle$ : GLDL group, $\bigcirc$ : GLDL-OX group. Each group : $n=5,{ }^{*}$ : significant difference $(p<0.05)$

difference of SMC number between the groups of $n L D L$ and GLDL (Fig. 1).

\section{SMC migration rate}

The migrated SMC number in the GLDL-LOX group was significantly increased compared with the nLDL, GLDL and GLDL-OX groups. This suggested that GLDL-LOX might promote SMC migration. There was no significant alteration by the addition of GLDL-OX, compared with the nLDL group (Fig. 2).

\section{PDGF-R immunoreactivity}

SMC in the nLDL and GLDL-LOX groups showed immunoreactivity of PDGF-R in the cell surface and cytoplasm (Fig. 3). The GLDL-LOX group revealed a higher number of PDGF-R positive cells than the $\mathrm{nLDL}$ group $(p<0.05)$. PDGF-R expression was not induced by the procedure of oxidation of glycated LDLs in cultured SMC (Fig. 4).

\section{Apoptosis and Bax protein}

Apoptotic cells, detected by the TUNEL method, and Bax protein-positive cells were demonstrated in SMC of

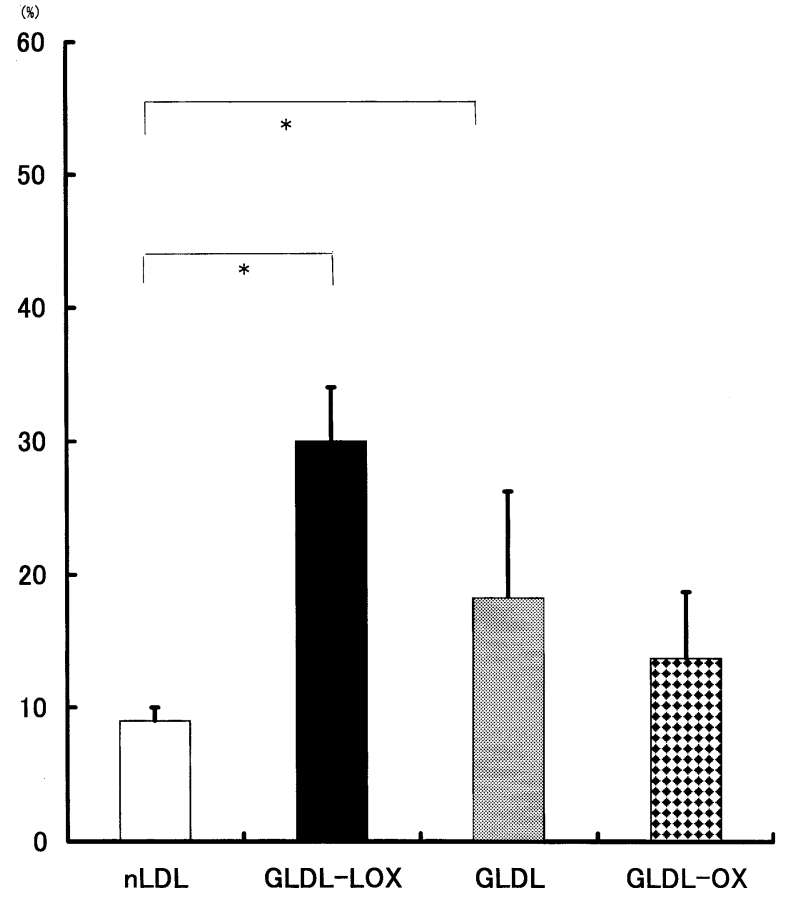

Fig. 2. This bar graph showing the rate of migrated SMC after incubation. The value of GLDL-LOX group is higher than $\mathrm{nLDL}$ (control) group. Each group : $n=5,{ }^{*}$ : significant difference $(p<0.05)$

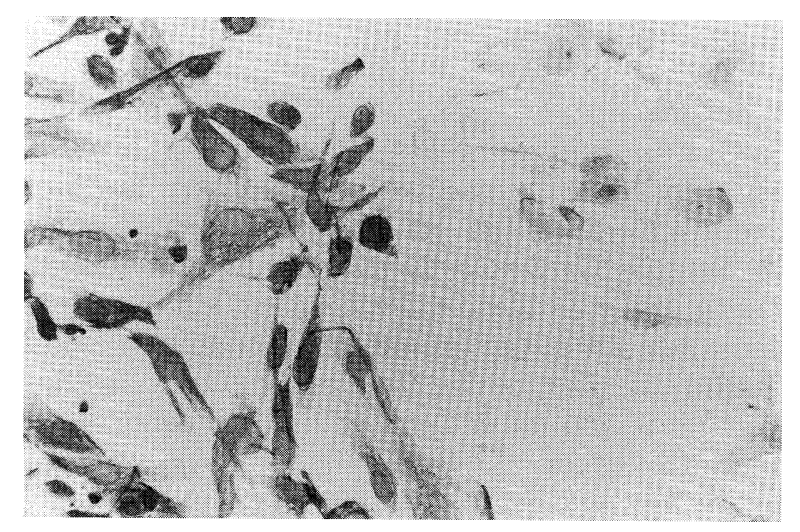

Fig. 3. This photograph revealing PDGF-R positive SMC of GLDL-LOX group. Intense immunoreactivity is demonstrated in the SMC cytoplasm, left side of this photograph (original magnification $\times 200$ ).

the GLDL and GLDL-OX groups (Fig. 5). The number of apoptotic cells of GLDL was significantly higher than that of the GLDL-OX group. No notable reactivity was found in SMC of the nLDL and GLDL-LOX groups, for either apoptosis or Bax protein (Figs. 6, 7).

\section{Protease activity}

In the GLDL-LOX group, protease activity, calculated by the formula described above, was significantly higher than in the nLDL, GLDL and GLDL-OX groups (Fig. 8). This 


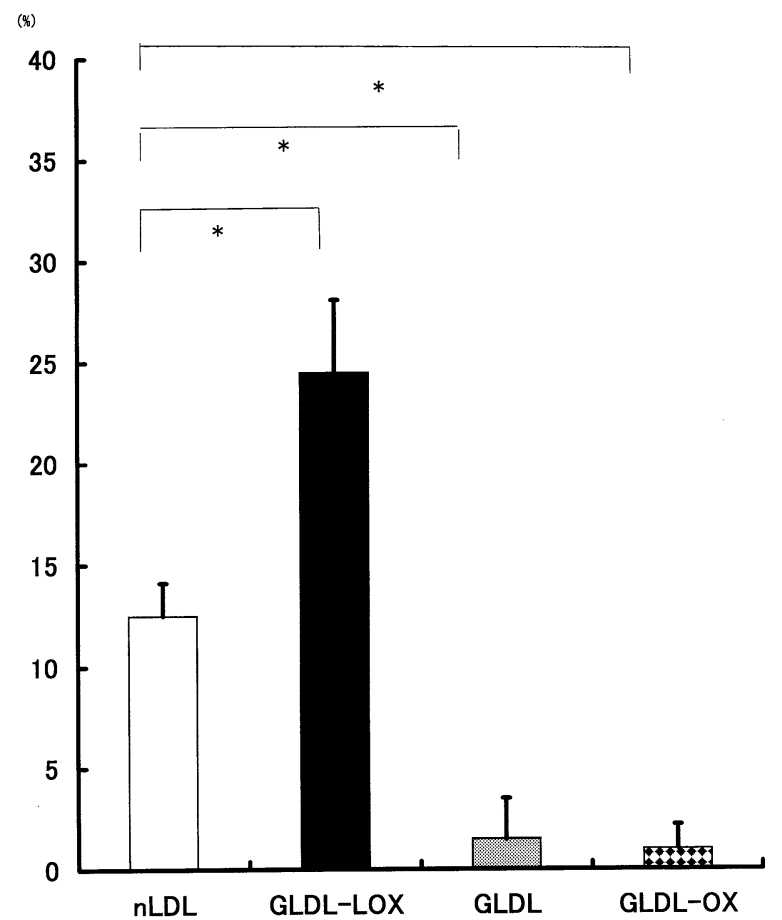

Fig. 4. This bar graph showing the rate of positive $S M C$ for PDGF-R on immunohistochemistry. The value of GLDLLOX group is higher than nLDL (control) group. In contrast, the values of GLDL and GLDL-OX are lower than control. Each group : $n=5,{ }^{*}$ : significant difference $(p<0.05)$

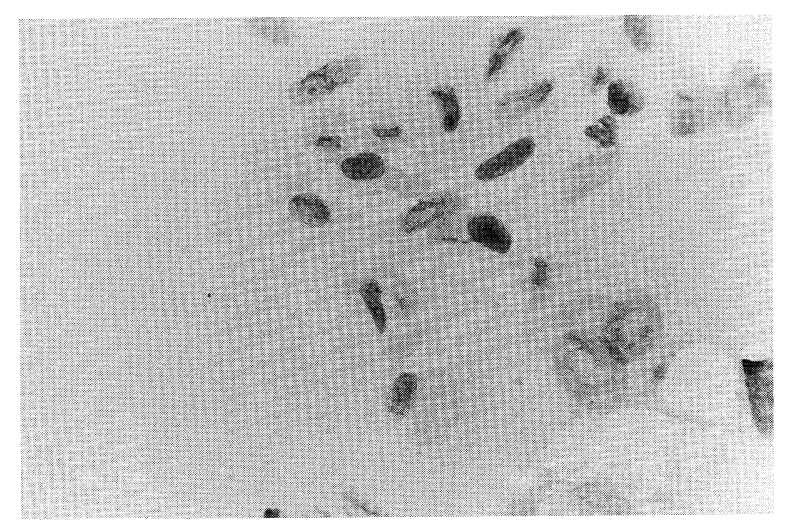

Fig. 5. This photograph revealing apoptotic SMC of GLDL group, detected by TUNEL method. The nuclei also show shrinking appearance (original magnification $\times 200$ ).

suggested that GLDL-LOX evoked the secretion of protease from SMC, leading to the casenolytic activity.

\section{Discussion}

There was the evidence that the biochemical characteristics of glycated LDL are similar to those of oxidized LDL. Actually, superoxide anions were produced in a hyperglycemic state, followed by the formation of oxidized LDL (12). By lipid analyses of glycated LDL, LDL incubat-

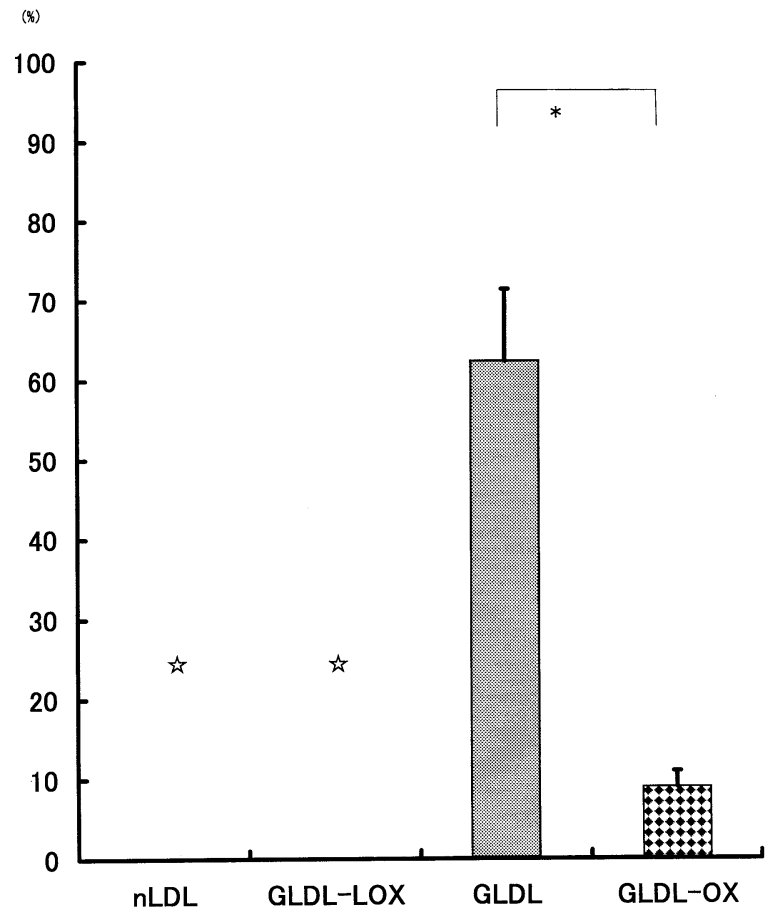

Fig. 6. This bar graph showing the rate of apoptotic SMC detected by TUNEL method. Only GLDL and GLDL-OX groups reveal apoptotic SMC. The value of GLDL group is higher than GLDL-OX group. it: no expression of apoptosis, * : significant difference $(p<0.05)$

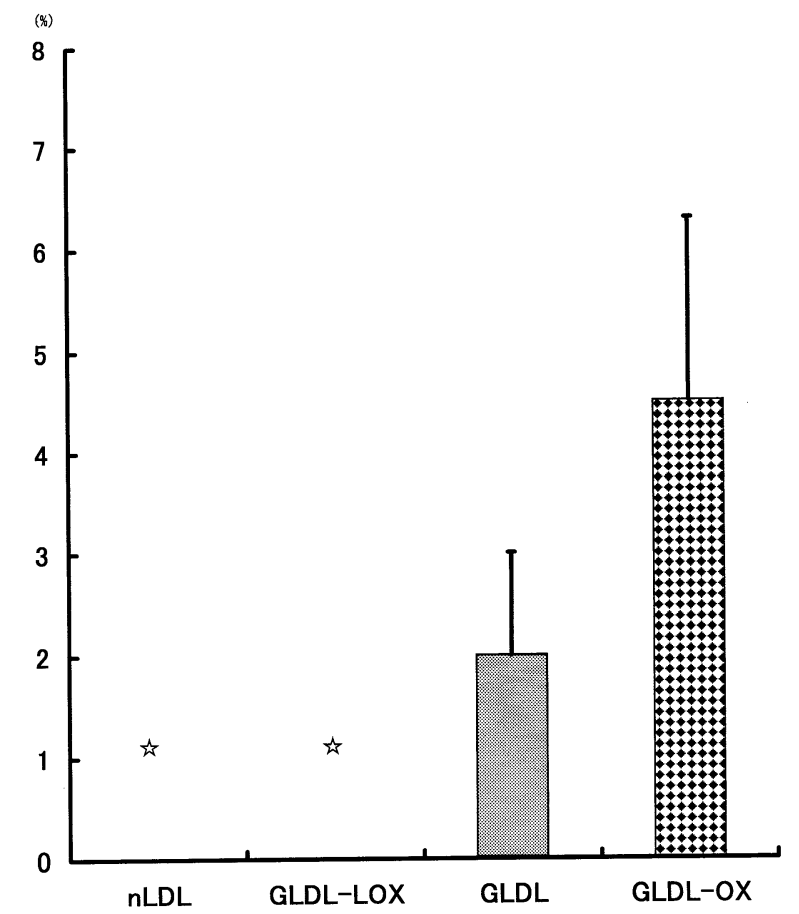

Fig. 7. This bar graph showing the rate of positive SMC for Bax protein. Only GLDL and GLDL-OX groups reveal expression. Each group: $n=5$, No significant difference is noted. is: no expression of Bax protein. 


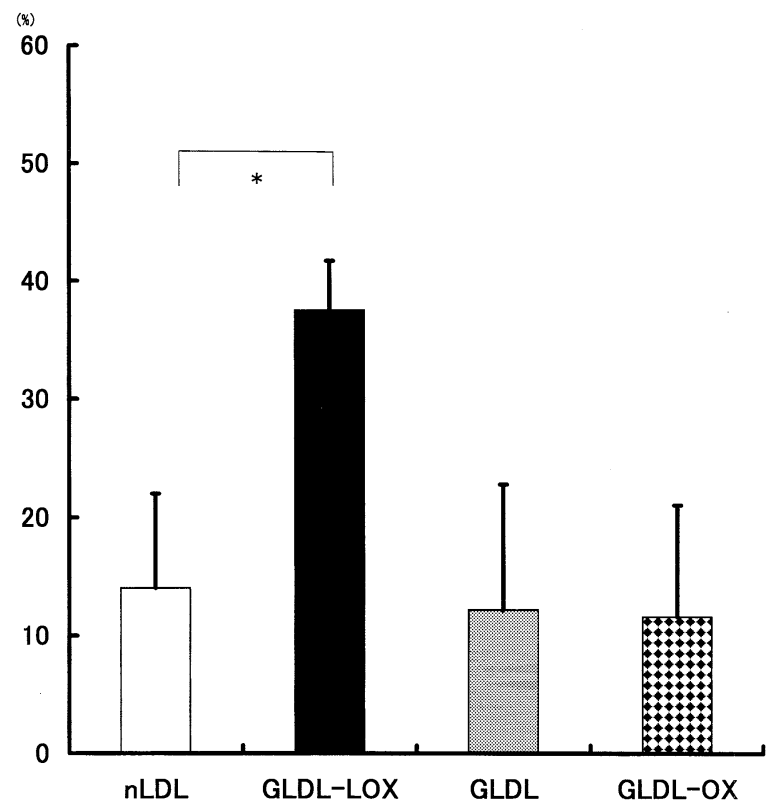

Fig. 8. This bar-graphical presentation shows the protease activity of each group after 1 month reaction. The value of GLDL-LOX group is higher than nLDL (control) group. Each group : $n=5,{ }^{*}$ : significant difference $(p<0.05)$

ed with glucose and $1 \mathrm{mM}$ EDTA was described as primary surface oxidized LDL, characterized by phosphatidylcholine hydroperoxide production $(13,14)$. Thus, GLDL was described as glycated and auto-oxidized LDL (15). The GLDL-LOX utilized for this study was not determined exactly as "pure glycated, non-oxidized LDL" because it was unclear whether BHT and EDTA could inhibit oxidation completely, especially minimal oxidation. Minimal oxidation could not be evaluated by the TBARS method. Although further investigation for lipid analysis might be required, our GLDL-LOX could be described as slightly-oxidized LDL.

In our results, GLDL-LOX addition increased number of the SMC and migrated SMC. The cell number increasing could not conclusively indicate SMC proliferation, but Ki67 expression was demonstrated in a few SMC of GLDL-LOX (data not shown). Thus, these results suggest that glycated LDL evokes intimal thickening by migrated SMC proliferation in the early stage of diabetes mellitus ; the state of little oxidative stress. PDGF receptor, already associated with the expression of SMC in thickened intima of atherosclerosis (16), could be related to this proliferation by our method of counting the positive cell number. In addition, GLDL-LOX could induce the phenotypic change of SMC, leading to SMC migration. In contrast, GLDL-OX and GLDL revealed strong cell injury or apoptosis induction. These results might imply the formation of a necrotic core in late or advanced stage atherosclerosis.

Two different types of glycation-reactive substance are known. One is the amadori product, produced in the early stage during glycation, approximately one to three weeks after conmencement (17). The other is advanced glycosylation end products (AGE), formed in the late stage (18). In this study, little attention was paid to AGE, because the time of glycation was almost 1 week. Thus, the amadori product might have played a major role in some of our results. The function of the amadori product has not been investigated, but its existence was established in experimental diabetic rat aorta (19).

Apoptosis, well-known as the morphosis of programmed cell death, plays a key role in atherosclerosis (20). Oxidative stress is a mediator of apoptosis (21). Our study suggested that LDL glycation itself caused neither apoptosis nor necrosis, with little oxidation.

In glycated LDL, apoB protein glycation, mainly on lysine residues, and the change of lipid composition were main alterations in addition to auto-oxidation (22). Glycated LDL enhances uptake of LDL by monocytederived macrophages. This is probably mediated by a low-affinity, high capacity surface receptor (called AGE receptor) (23) or scavenger receptor (24). Glycation and oxidation are closely related and could mutually accelerate each other (25). Oxidized LDL performs various functions (26), and the combined glycation and oxidation of LDL generated a product that was more atherogenic than either glycation or oxidized LDL alone (27).

The importance of atheromatous plaque rupture in the incidence of acute myocardial infarction has been identified (28). Protease, like matrix metalloprotease (MMP), took a key role of plaque rupture in the extracellular matrix degradation. The expression of MMP has been demonstrated on migrated SMC in the ruptured atheroma (29-31). Although we did not investigate the function of MMP in detail, the casenolytic activity we presented could suggest MMP-3 (stromelysin) activity (11). Thus, this result might suggest that diabetic patients are at risk of plaque rupture.

We emphasized on the clear difference among glycated slightly-oxidized, glycated, auto-oxidized, and glycated extensively-oxidized LDLs. Further investigation will be required for detail biochemical or molecular analyses, but our hypothesis was that LDL glycation itself promoted the early atherosclerosis and glycated LDL was concerned with the formation of a necrotic core under the additional oxidative stress. It was concluded that a high level of serum glucose was a definitive high risk factor for atherosclerosis, even with little oxidative stress.

Acknowledgments : The authors wish to appreciate Professor Isamu Sakurai and Professor Norimichi Nemoto, Department of Pathology, Nihon University School of Medicine, for their advice and revision of the manuscript in this research. 


\section{References}

(1) Keen $\mathrm{H}$ and Jarret $\mathrm{HJ}$ : The WHO multinational study of vascular disease in diabetes. Diabetes Care, 2 : 187195, 1979

(2) Wissler RW, Hiltscher L, Oinuma T, and PDAY Research Group: The lesions of atherosclerosis in the young; from fatty streak to intermediate lesion. In: Atherosclerosis and Coronary Artery Disease. pp 475-479, Lippincott-Raven Press, 1996

(3) Steinbrecher UP, Parthasarthy S, Carew TE, Khoo JC, Witztum JL, and Steinberg D: Modification of low density lipoprotein by endothelial cell involves lipid peroxidation and degradation of low density lipoprotein phospholipid. Proc Natl Acad Sci USA, 81 : 3883-3887, 1984

(4) Steinbrecher UP, Zhang $H$, and Lougheed M: Role of oxidatively modified LDL in atherosclerosis. Free Radic Biol Med, 9 : 155-168, 1990

(5) Browlee $M$ : Glycation and diabetic complications. Diabetes, 43: 836-841, 1994

(6) Cohen MP, Lautenslager G, and Shea E : Glycated LDL concentrations in non-diabetic and diabetic subjects measured with monoclonal antibodies reactive with glycated apolipoprotein B epitopes. Eur J Clin Chem Biochem, 31: 707-713, 1993

(7) Kobayashi K, Watanabe J, Umeda F, and Nawata H: Glycation accelerates the oxidation of low density lipoprotein by copper ions. Endocrinol J, 42: 461-465, 1995

(8) Picard S: Lipoprotein glyco-oxidation. Diabetes Metab, 21: 89-94, 1995

(9) Hunt JV, Smith CCT, and Wolff SP: Auto-oxidative glycosylation and possible involvement of peroxides and free radicals in LDL modification of glucose. Diabetes, 39: 1420-1424, 1990

(10) Lopes-Virella MF, Klein RL, Lyons TJ, Stevenson HC, and Witztum JL: Glycosylation of low-density lipoprotein enhances cholesteryl ester synthesis in human monocyte-derived macrophages. Diabetes, $37: 550-$ 557,1988

(11) Knox JB, Sukhova GK, Whittmore AD, and Libby $P$ : Evidence for altered balance matrix metalloproteases and their inhibitors in human aortic diseases. Circulation, 95: 205-212, 1997

(12) Kimura H, Minakami H, and Kimura S: Release of superoxide radicals by mouse macrophage stimulated by oxidative modification of glycated low density lipoproteins. Atherosclerosis, 118: 1-8, 1995

(13) Kamido $\mathrm{H}$ : How does glycation promote oxidation? Doumyakukouka, 24 : 97-106, 1996 (in Japanese)

(14) Graier WF, Simecek S, Kukovetz WR, and Kostner GM. High-D-glucose-induced changes in endothelial $\mathrm{Ca}^{2+}$ / EDRF signaling is due to generation of superoxide anions. Diabetes, $45:$ 1386-1395, 1996

(15) Hunt JV, Dean RT, and Wolff SP : Hydroxyl radical production and autooxidative glycosylation; glucose autooxidation as the cause of protein damage in the experimental glycation model of diabetes mellitus and aging. Biochem J, 256: 205-212, 1988

(16) Krettek A, Fager G, Jernberg P, Ostergren-Lungden G, and Lustig $F$ : Quantitation of platelet-derived growth factor receptors in human arterial smooth muscle cells in vitro. Arterioscler Thromb Vasc Biol, 17: 2395-2404, 1997

(17) Bucala R and Cerami A: Advanced glycosylation: Chemistry, biology, and implications for diabetes and aging. Adv Phramacol, 23 : 1-34, 1992

(18) Palinski W, Koschinsky T, and Butler SW : Immunological evidence for the presence of advanced glycosylation end products in atherosclerotic lesions of euglycemic rabbits. Arterioscler Thromb Vasc Biol, 15 : 571-582, 1995

(19) Sakata N, Meng J, Miyamoto K, Takebayashi S, Furukawa $\mathrm{T}$, and Nakajima $\mathrm{K}$ : Vascular biology of diabetic macroangiopathy: role of nonenzymatic glycation of extracellular matrix in atherogenesis. Doumyakukouka, 24: 107-115, 1996 (in Japanese)

(20) Geng YJ: Regulation of programmed cell death or apoptosis in atherosclerosis. Heart Vessels, $12: 76-80$, 1997

(21) Buttke TM and Sandstorm PA: Oxidative stress as a mediatory of apoptosis. Immunol Today, 15: 7-20, 1994

(22) Sobenin IA, Tertov VV, and Orekhov AN : Atherogenic modified LDL in diabetes. Diabetes, 45(suppl 3) : 3539, 1996

(23) Vlassara $\mathrm{H}$, Brownlee $\mathrm{M}$, and Cerami A : Nodal macrophage receptor for glucose-modified proteins is distinct from previously described scavenger receptor. J Exp Med, 164 : 1301-1309, 1986

(24) Dobrian A, Lazar V, Tirziu D, and Simionescu M: Increased macrophage uptake of irreversibly glycated albumin modified-low density lipoproteins of normal and diabetic subjects is mediated by non-saturable mechanisms. Biochem Biophys Acta, 1317: 5-14, 1996

(25) Bucala R, Makita Z, Kochinsky T, Cerami A, and Vlassara $\mathrm{H}$. Lipid advanced glycosylation: Pathway for lipid oxidation in vivo. Proc Natl Acad Sci USA, 90 : 6434-6438, 1993

(26) Oinuma T, Yamada T, and Sakurai I: Effects of copperzinc type superoxide dismutase on the proliferation and migration of cultured vascular smooth muscle cells induced by oxidized low density lipoprotein. J Atheroscler Thromb, $4:$ 79-84, 1997

(27) Baynes JW : Role of oxidative stress in development of complications in diabetes. Diabetes, $40: 405-412,1991$

(28) Davis $\mathrm{MJ}$ and Thomas AC : Plaque fissuring-the cause of acute myocardial infarction, sudden ischemic death, and crescendo angina. $\mathrm{Br}$ Heart J, $50: 127-134,1983$

(29) Libby $\mathrm{P}$ : The molecular bases of the acute coronary syndromes. Circulation, 91: 2844-2850, 1995

(30) Schonbeck U, Mach F, Sukhova GK, Murphy C, Bonnefoy JY, Fabunmi RP, and Lippy P : Regulation of matrix metalloprotease expression in human vascular smooth muscle cells by $T$ lymphocytes; a role for CD40 signaling in plaque rupture. Circ Res, 81: 448-454, 1997

(31) Rajavashisth TB, Xu XP, Jovinge $S$, Meisel $S, X u X O$ Chai NN, Fishbein MC, Kaul S, Cercek B, Sharifi B, and Shan PK: Membrane type 1 matrix metalloproteases expression in human atherosclerotic plaque; evidence for activation by proinflammatory mediators. Circulation, 99 : 3103-3109, 1999 